\title{
Erratum
}

\section{The Uterus Sustains Stable Biological Clock during Pregnancy}

\author{
Shizuko Akiyama, ${ }^{1,2}$ Hidenobu Ohta, ${ }^{1,2,3 *}$ Shimpei Watanabe, ${ }^{1,2}$ Takahiro Moriya, ${ }^{4}$ \\ Aya Hariu, ${ }^{4}$ Norimichi Nakahata, ${ }^{4}$ Hiroshi Chisaka, ${ }^{1,3}$ Tadashi Matsuda, ${ }^{1,2}$ \\ Yoshitaka Kimura, ${ }^{5}$ Shigeru Tsuchiya, ${ }^{2}$ Hajime Tei, ${ }^{6}$ Kunihiro Okamura ${ }^{1,3}$ and \\ Nobuo Yaegashi, ${ }^{1,3}$
}

\footnotetext{
${ }^{1}$ Center for Perinatal Medicine, Tohoku University Hospital, Sendai, Japan ${ }^{2}$ Department of Pediatrics, Tohoku University Hospital, Sendai, Japan

${ }^{3}$ Department of Obstetrics and Gynecology, Tohoku University Hospital, Sendai, Japan

${ }^{4}$ Department of Cellular Signaling, Graduate School of Pharmaceutical Sciences, Tohoku University, Sendai, Japan

${ }^{5}$ Tohoku University Institute for International Advanced Research and Education, Sendai, Japan

${ }^{6}$ Kanazawa University Institute of Science and Engineering Faculty of Natural System, Kanazawa, Japan
}

Tohoku J. Exp. Med., 221: 287-298, 2010.

In the version of this article published in the August issue, 2010, the affiliation number of the tenth author (Shigeru Tsuchiya) was mistyped (page 287). The correct affiliation is shown above. In addition, the lettering of ' $\mathrm{ZT}$ ' is a misprint for 'CT' in panels A, B and C of Figures 6 and 7 (pages 294 and 295).

A

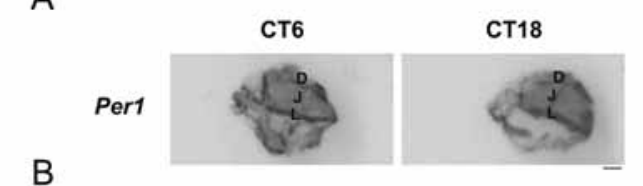

B

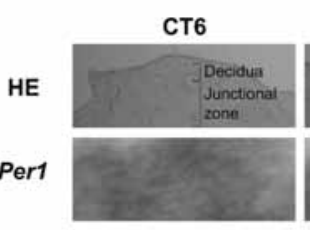

C

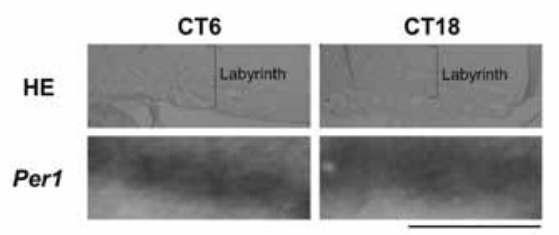

D

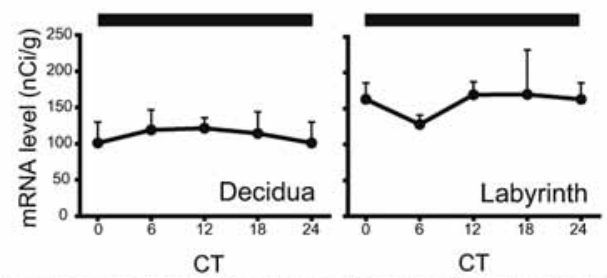

Fie. a. The mRNA expression profiles of Perl gene in the placenta from wild-type rats in cuastant dankness (DD) on E12 (A) The unimals were exposed to DD for 2 consecutive days prior to explants to elininate the lighting effects an the biologi-
al clack Representative in

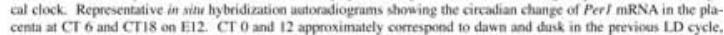

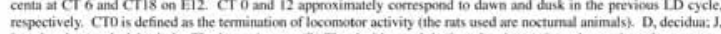
jubctional zone: $L$ L labyrinth. The bur $=1$ mim. (B) The decidua and the junctional zone in a placental sectioe counter-

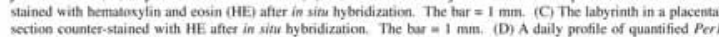

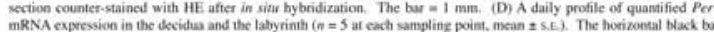

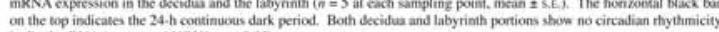
on the top indicates the 24 . h continuous dark,
in Per/ mRNA
A

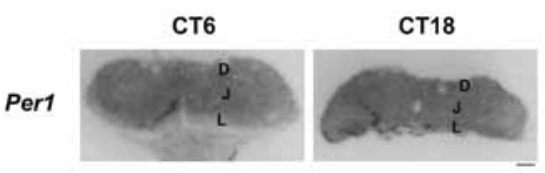

B

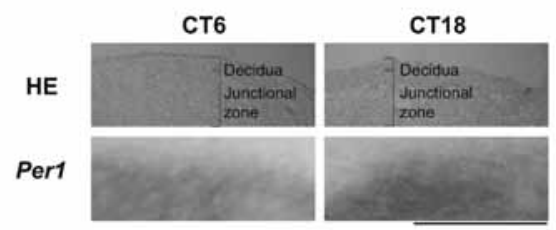

C

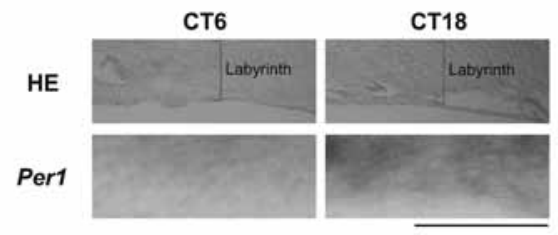

D

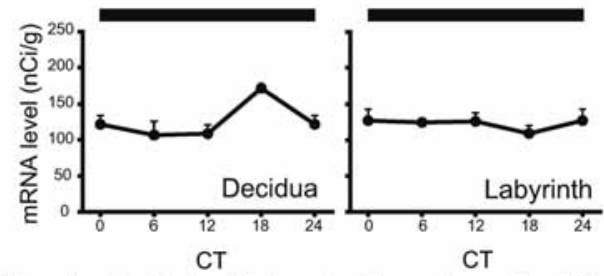

ig. 7. The mRNA expression profiles of $\mathrm{Per} / \mathrm{gene}$ in the placenta from wild-type rats in constant darkiness (DD) on E22. The animals were exposed to DO for 2 consectulive hyys prior to explants to eliminate the lighting effects on the tiologi-

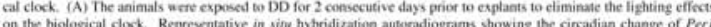

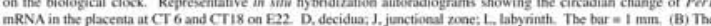
decidua and the junstionat zone in a placemal section counter-stained with bematoxylin and cosin (HE) affer in sise bybridization. The har $=1 \mathrm{~mm}$. (C) The labyrinth in a placental section countet-stained with HE after in simu bybridiza:

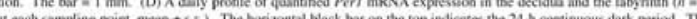

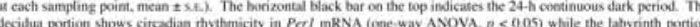
shows no ritythicity (Ooe way ANOVA. $p>005$ ) 